\title{
Title: Comment on Cesana-Arlotti et al. (2018)
}

\author{
Authors: Masoud Jasbi ${ }^{1 *}$, Manuel Bohn ${ }^{2,3}$, Bria Long ${ }^{2}$, Abdellah Fourtassi ${ }^{2}$, David Barner ${ }^{4}$, \\ Michael C. Frank ${ }^{2 *}$
}

\begin{abstract}
Affiliations:
${ }^{1}$ Department of Linguistics, Harvard University, Cambridge, MA, USA. ${ }^{2}$ Department of Psychology; Stanford University, Stanford, CA, USA. ${ }^{3}$ Leipzig Research Center for Early Child Development; Leipzig University, Leipzig, Germany. ${ }^{4}$ Department of Psychology, University of California San Diego, San Diego, CA, USA.

*Correspondence to: masoud_jasbi@,fas.harvard.edu, mcfrank@stanford.edu
\end{abstract}

\begin{abstract}
:
The origins of logical concepts is one of the central topics in cognitive science. Cesana-Arlotti and colleagues provide novel eye-tracking measures from preverbal infants compatible with disjunctive reasoning. However, the evidence is not conclusive. We provide a simpler object tracking account that would produce the same processing signatures. Future research must make a priori predictions that distinguish these accounts.
\end{abstract}

\section{Main Text:}

Are primitive logical and inferential abilities the result of a protracted developmental process or are they part of the innate fabric of a uniquely human mind and present in infancy prior to the emergence of language? Cesana-Arlotti et al. address this question by tracking eye-movements of 12- and 19-month-old infants in a violation of expectation task $(1,2)$. They report that infants look longer at scenes that violate the conclusions of disjunctive syllogistic reasoning, compared to scenes that do not. More importantly, they report signatures of increased processing during the period in which deductive reasoning is hypothesized to take place. These experiments are clever and indeed compatible with deductive inference. But the reported findings are also compatible with an account of object-tracking that does not involve logical operations. Therefore, stronger evidence is needed to support a role for logical reasoning in these tasks.

In Experiments 1 and 2, two objects (e.g., flower and dinosaur), were hidden in two locations: behind an occluder and inside a cup (Figure 1, original paper). Next, the occluder was removed and the object behind it (e.g., the dinosaur) was revealed. In "consistent" trials the content of the cup was revealed to be the flower, but in "inconsistent" trials it was the dinosaur. Infants showed increased looking time when the cup contained the dinosaur, suggesting that they did not expect this outcome. This result is compatible with deductive reasoning in infants, but - as the authors point out - infants may have looked longer when the same object was revealed twice in two different locations (as happened in the inconsistent trials). To rule out this explanation, in Experiments 3 and 4 the content of the cup was never revealed. However, Experiments 1-4 could also be explained by infants' object tracking abilities $(3,4)$. Rather than deductive inference, infants may have tracked the number of objects and their locations. When one of two objects is 
removed from behind the occluder, the subsequent emergence of two distinct objects from the same location is surprising.

Experiments 5 and 6 provide the novel and promising contribution of this paper that could potentially address this leaner explanation. In these Experiments, infants were presented with a set of trials identical to those in Experiments 3 and 4, except that now the content of the cup was already known. Cesana-Arlotti et al. reasoned that if Experiments 3 and 4 engage deductive inference then infants should exhibit different processing signatures than in Experiments 5 and 6 in which inference cannot play a role. To test this, they compared the putative inference conditions (Experiments $3 \& 4$ ) to the no-inference conditions (Experiments $5 \& 6$ ) with respect to three oculomotor measures in the "potential deduction phase" (during the appearance of the disambiguating object): (1) horizontal fixation (mean x position), (2) object-to-cup gaze shifts, and (3) pupil dilation. They reported that during this period, infants showed a significant increase in all three measures. They argued that these results support disjunctive reasoning in infants.

While these results are intriguing, they do not weigh unambiguously in favor of disjunctive syllogism. In Figure 1, we describe an object-tracking account that relies only on infants updating their mental models of the scenes and keeping them consistent. In scene (i), infants begin with the knowledge that two objects are present in the scene (e.g., snake and ball). Then, in scene (ii), the objects disappear behind the occluder and their location is updated in the mental model. In scene (iii), infants see a cup go behind the occluder and return with an unknown object inside. Representing scene (iii) requires revision to the previous model: given that only one object is inside the cup, it can't be that both objects are behind the occluder. Therefore the model is minimally revised to maintain consistency: the locations of the objects are no longer known. In scene (iv), infants see the snake come out from and then go back behind the wall; this scene thus provides evidence that the snake is behind the occluder. Given object permanence, now infants can resolve the unknown location of the other object and derive a consistent model with respect to the entity and location of the objects. This process of model update predicts the same outcome as the syllogistic account (Figure 1, right branch). And critically, it also involves computations during the "potential deduction phase" which like a syllogistic inference could explain reported differences in infant gaze measures.

Above we offered one possible alternative to Cesana-Arlotti et al's account. A more general version of our concern is that the authors do not articulate the null hypothesis - and its predictions - against which their proposal should be evaluated. A clean test of the syllogistic reasoning account requires developing clear predictions from the leaner accounts and a method that rules out these leaner reasoning processes (5). While Cesana-Arlotti et al's work develops new and promising measures, their work also illustrates some of the perils that come with richer, more sophisticated methods: because of the proliferation of possible analyses, it is critical to pre-register specific predictions of different accounts to avoid false positives $(\sigma)$. Otherwise, specific analytic decisions can appear post-hoc; for example, the use of different tests to take into account the autocorrelated nature of the eye-tracking data, the use of mean horizontal fixation (mean $\mathrm{x}$ ) as a processing measure, or the decision to consolidate age groups in the gaze shift analysis (an analysis which is non-significant for the youngest group alone). 
In sum, Cesana-Arlotti et al. argue for innate logical representations that underlie object tracking by looking deeper into subtle aspects of infant looking behavior. However, the effects they report are also potentially compatible with simpler processes, which might be shared with other animals. Building on their work, researchers now must find paradigms that make a sharper distinction between the predictions of disjunctive syllogism and simpler object tracking accounts that do not assume logical operations. Only then will we be able to distinguish linguistic and nonlinguistic knowledge of logical concepts.

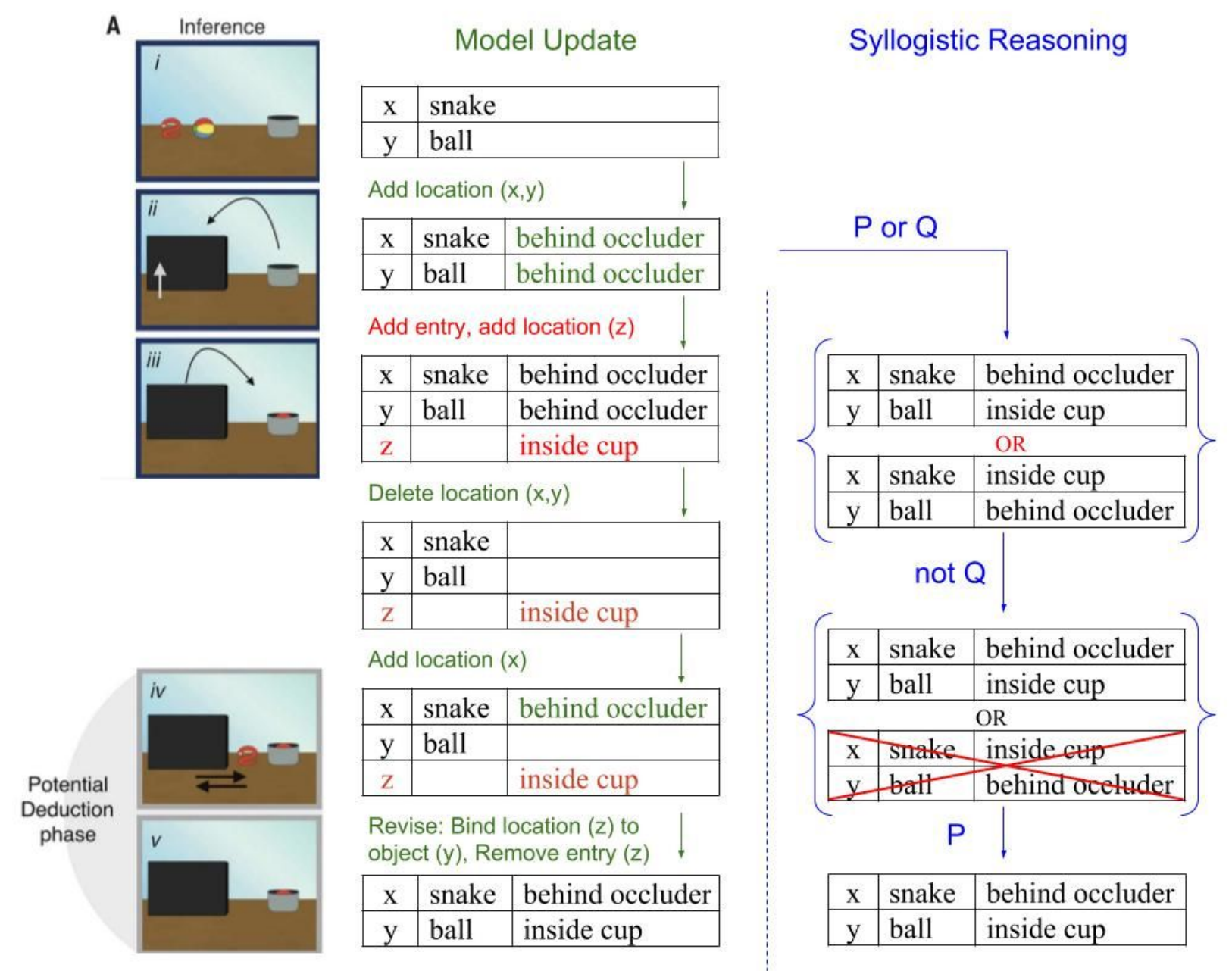

Fig. 1. Representational steps for "Model Update" and "Syllogistic" accounts in the scenes (i-v) of the inference condition, experiments 3 and 4. "Model Update" uses operations "Add" which adds relevant information from the visual scene, "Delete" which removes information from the working memory, and "Bind" which binds entries and prunes the model to keep it consistent, e.g. with object permanence. "Syllogistic reasoning" uses the logical operations "or" (disjunction) and "not" (negation). 


\section{Acknowledgments:}

We wish to thank Susan Carey and Roman Feiman for their insightful comments.

\section{References:}

[1] Cesana-Arlotti, N., Martín, A., Téglás, E., Vorobyova, L., Cetnarski, R., \& Bonatti, L. L. (2018). Precursors of logical reasoning in preverbal human infants. Science, 359(6381), 1263-1266.

[2] Baillargeon, R., Spelke, E., \& Wasserman, S. (1985). Object permanence in five-month-old infants. Cognition, 20, 191-208.

[3] Wynn, K. (1992). Addition and subtraction by human infants. Nature, 358(6389), 749.

[4] Xu, F., \& Carey, S. (1996). Infants' metaphysics: The case of numerical identity. Cognitive psychology, 30(2), 111-153.

[5] Mody, S., Carey S. (2016) The emergence of reasoning by the disjunctive syllogism in early childhood. Cognition, 154, 40-48.

[6] Nosek, B. A., Ebersole, C. R., DeHaven, A. C., \& Mellor, D. T. (2018). The preregistration revolution. Proceedings of the National Academy of Sciences, 201708274. 